\title{
Inhibition of mTOR sensitizes breast cancer stem cells to radiation-induced repression of self-renewal through the regulation of MnSOD and Akt
}

\author{
YUANHUI LAI ${ }^{1 *}$, XINPEI YU ${ }^{2,3^{*}}$, XIAOHONG LIN ${ }^{1}$ and SHANYANG HE ${ }^{4}$ \\ ${ }^{1}$ Department of Thyroid and Breast Surgery, The First Affiliated Hospital of Sun Yat-sen University, \\ Guangzhou, Guangdong 510700; ${ }^{2}$ Department of Geriatric Infection and Organ Function Support Laboratory, \\ Guangzhou General Hospital of Guangzhou Military Command, Guangzhou, Guangdong 510010; \\ ${ }^{3}$ The Geriatric Ward, Guangzhou General Hospital of Guangzhou Military Command, Guangzhou, \\ Guangdong 510010; ${ }^{4}$ Department of Obstetrics and Gynecology, The First Affiliated Hospital, \\ Sun Yat-sen University, Guangzhou, Guangdong 510700, P.R. China
}

Received July 24, 2015; Accepted December 9, 2015

DOI: $10.3892 / \mathrm{ijmm} .2015 .2441$

\begin{abstract}
The sensitization of breast cancer stem cells (BrCSCs) to the inhibitive effects of radiotherapy through adjuvant therapy which targets oncogenic pathways represents a prospective strategy for improving the effect of radiation in patients with triple-negative breast cancer (TNBC). Mammalian target of rapamycin (mTOR) activation is one of the most frequent events in human malignancies, and is critical for sustaining the self-renewing ability of cancer stem cells (CSCs); inhibition by rapamycin is an effective and promising strategy in anticancer treatments. In the present study, we found that mTOR activity was closely related to the self-renewal ability of BrCSCs, and in triple negative MDA-MB-453 and MDA-MB-468 cells, rapamycin repression of mTOR phosphorylation decreased the number of mammospheres and helped to sensitize the resistant CSCs to low-dose radiation therapy. By inhibiting $\mathrm{mTOR}$ and mitochondrial manganese superoxide dismutase (MnSOD), we confirmed that rapamycin functioned through the mTOR/MnSOD/reactive oxygen species (ROS) signaling pathway, and the existence of Akt governed the rapamycin-induced asymmetric division (AD) of stem cells in cases of radiation-treated breast cancer. The synergic effects of rapamycin and low-dose radiation induced the $\mathrm{AD}$ of stem cells, which then resulted in a decrease
\end{abstract}

Correspondence to: Professor Shanyang He, Department of Obstetrics and Gynecology, The First Affiliated Hospital of Sun Yat-sen University, 58 Zhongshan Second Road, Guangzhou, Guangdong 510700, P.R. China

E-mail: prof_hsy5g777@163.com

${ }^{*}$ Contributed equally

Key words: radiotherapy, rapamycin, mammalian target of rapamycin, manganese superoxide dismutase, reactive oxygen species, Akt, triple-negative breast cancer, cancer stem cells in the number of mammospheres, and both were mediated by MnSOD. Governed by Akt, the consequent inhibition of ROS formation and oxidative stress preserved the AD mode of stem cells, which is critical for an improved radiotherapy response in clinical treatment, as the tumor group is thus easier to eliminate with radiation therapy. We posit that an in-depth understanding of the interaction of radiation with CSCs has enormous potential and will make radiation even better and more effective.

\section{Introduction}

Worldwide, breast cancer is the leading type of cancer in women, and is much more common in developed countries, due to greater wealth and related dietary habits. Long-term use of oral contraceptives and low body mass index (BMI) are associated with an increased risk of premenopausal breast cancer $(1,2)$. Breast cancer in young women is thought to be associated with high-grade tumors, negative hormone receptors and overexpression of human epidermal growth factor receptor 2 (HER2) (3). The overall worldwide burden of breast cancer has increased significantly, with the mortality rates steadily decreasing, owing to early detection and improved therapies (3). Survival rates are higher in the developed world, with nearly $80 \%$ of affected patients in England and the United States surviving for at least 5 years; however, in developing countries, survival rates are poorer (4-6).

Mammalian target of rapamycin (mTOR) plays a central role in the regulation of cell fate and cancer progression $(7,8)$. In particular, mTOR activation is one of the most frequent events in human malignancies, and inhibition of mTOR by rapamycin is an effective and promising strategy in anticancer treatments. mTOR activity is also critical for sustaining the self-renewal ability of cancer stem cells (CSCs) (9-11). mTOR inhibition is known to protect normal oral epithelial cells from radiation-induced epithelial stem cell depletion via the increased expression of manganese superoxide dismutase (MnSOD/SOD2), suggesting that interaction occurs between mTOR and MnSOD. MnSOD is a 
nuclear-encoded mitochondrial antioxidant enzyme, which is essential for the removal of superoxide radicals and governs the types of reactive oxygen species (ROS) egressing from the organelle (12), the accumulation of which damage DNA and the mitochondrial membrane, leading to tumorigenesis. The aberrant expression of MnSOD has been implicated in carcinogenesis and tumor resistance to therapy $(13,14)$; however, its roles in CSCs are still poorly understood.

Tumor groups are composed of heterogeneous cancer cells, of which the CSCs account only for a small population although they are crucial for tumorigenesis and treatment resistance. The CSCs are thought of as the roots of cancer, have low proliferative status and slow cell cycles, and remain steady throughout chemo-radiotherapy. Due to the negative response to major treatments, the elimination of CSCs has proven to be a key obstacle in curing cancer, and the existence of CSCs contributes to tumor relapse and resistance to clinical therapies $(11,15)$. The general perception is that CSCs are inherently resistant to radiation therapy, and this resistance is considered to be a general property of the stem cell group (11). However, diverse results have been detected in certain studies: on the one hand, CSCs have been found to be resistant to common chemo-radiotherapies, contributing to tumor occurrence and relapse (16-18); on the other hand, previous research has suggested that the tumorderived stem cells have different characteristics, and respond to radiotherapy in different ways (19). ROS activity is thought to be linked to the response to therapies: high levels of ROS are related to stronger productive properties of cancer cells, and are closely related to tumor recurrence and therapy resistance, whereas lower ROS levels are closely related to the signatures of CSCs (19-21).

Radiation is known to act as a powerful tool in the fight against breast cancer, and high doses of radiation are often used to eradicate tumor resistance to chemotherapies, acting as the last part of clinical treatments. However, studies have found that radiation increases therapy resistance by increasing the number of stem cells in cancer groups (22). On the one hand, radiation treatment can kill the majority of tumor cells, but, on the other hand, it can also transform cancer cells into treatment-resistant CSCs. The elimination of the majority of cancer cells paves the way for self-renewal of stem cells, making it more difficult to cure the tumor in the future (23). Controlling the radiation-resistant breast cancer stem cells (BrCSCs) during radiation treatment may ultimately improve curability and reduce the high radiation doses currently administered to breast cancer patients, and thus decrease acute and long-term side effects by decreasing the administration of high-dose radiation. The elimination of a smaller pool of breast CSCs in massive pools of cancer cells will eventually help to irradiate the remaining cancer cells, killing the cancer. We hypothesize that mTOR inhibition with rapamycin could then synergize with the antitumoral effects of radiation, which is one of the most frequent approaches in the treatment of triple-negative breast cancer (TNBC). Increased sensitization of tumors to radiotherapy will help to improve the prognosis of patients with breast cancer, particularly those patients with TNBC, which is more malignant and resistant to clinical therapies than other cancers. If we uncover the mechanisms through which the stem cells generate and transform, we may be able to block these happening and make the radiation therapy more powerful and less harmful.

\section{Materials and methods}

Cell culture, transfection and treatment. The human breast cancer cell lines SK-BR-3, T47-D, ZR-75-1, ZR-75-30, BT20, BT-549, MDA-MB-231, MDA-MB-453, MDA-MB-468, HCC1143 and HS-578T were all purchased from the Cell Bank of Shanghai Institute (Shanghai, China), and cultured in RPMI1640 medium (Gibco, Thermo Fisher Scientific, Waltham, MA, USA), containing 10\% fetal bovine serum (FBS) (Thermo Fisher Scientific) and $1 \%$ penicillin and streptomycin (Gibco, Thermo Fisher Scientific). The mammospheres (CSCs) were cultured in 1X DMEM/Ham's F12 medium, with $10 \mathrm{ng} / \mathrm{ml}$ epidermal growth factor (EGF), $10 \mathrm{ng} / \mathrm{ml}$ human basic fibroblast growth factor (hbFGF), $1 \mu \mathrm{g} / \mathrm{ml}$ hydrocortisone, $4 \mu \mathrm{g} / \mathrm{ml}$ insulin and $1 \%$ penicillin and streptomycin (Invitrogen, Carlsbad, CA, USA), as previously reported (24). Cancer cells were plated in ultra-low attachment dishes (Corning, Inc., Corning, NY, USA) to test their ability to form primary mammospheres in stem cell medium. On the 7th day, the number of mammospheres was counted as previously described (7,25-27). Briefly, a sphere is identified if it contains $>50$ cells, as was observed and counted under a microscope. The obtained mammospheres of different groups were disaggregated and then seeded into ultra-low attachment dishes to test their self-renewal ability for subsequent generation in continous culturation. Three individual pairs of siRNAs against mTOR and MnSOD and RFP-based shRNAs against Akt1 were all designed and synthesized by Gene Pharma (Shanghai, China). Transfection with siRNAs was performed using Lipofectamine 2000 (Invitrogen) according to the manufacturer's instructions. The cells were irradiated using a Cs-137 irradiator (GSM:GSR D1). Ionizing radiation was carried out in strict accordance with the clinical criteria. The cells were exposed to ionizing radiation prior to use in the experiments. Rapamycin (ab120224; Abcam, Shanghai, China) was used to inhibit mTOR activity, and was prepared at a concentration of $20 \mu \mathrm{M}$. An Akt inhibitor (Akti-1/2, ab142088; Abcam, Cambridge, MA, USA) was used to inhibit Akt phosphorylation.

Western blot analysis and immunofluorescence assay. For western blot analysis, proteins of different groups were harvested in RIPA lysis buffer (Beijing Biotech, Beijing, China), with protease/phosphatase inhibitor cocktail (100X, no. 5872; Cell Signaling Technology, Inc., Danvers, MA, USA), and subsequently subjected to $10 \%$ SDS-PAGE separation. Monoclonal or polyclonal anti-p-mTOR (Ser2481, no. 2974; Cell Signaling Technology, Inc.), anti-MnSOD (DD-17, no. S5069, Sigma-Aldrich, St. Louis, MO, USA) and anti- $\beta$-actin (no. 4967; Cell Signaling Technology, Inc.) were diluted to 1:1,000-1:5,000 for western blot analysis. HRP goat anti-mouse (no. 554002) and HRP goat anti-rat (no. 554017) were purchased from BD Pharmingen (San Diego, CA, USA).

For the immunofluorescence assay, the cells were planted in chambers and then fixed with $10 \%$ formalin for $15 \mathrm{~min}$. The cells were subsequently blocked in $2 \%$ normal goat serum (ab7481; purchased from Abcam, Cambridge, MA, USA), and incubated with the primary antibody (the same one used for western blot analysis) for $1 \mathrm{~h}$ in PBST, and sequentially with goat anti-rabbit or goat anti-mouse secondary antibody for a least 30 min with Alexa Fluor ${ }^{\circledR} 488,568$ or 633 dye; finally, the cells were incubated for $15 \mathrm{~min}$ with DAPI nuclear 
$\mathbf{A}$
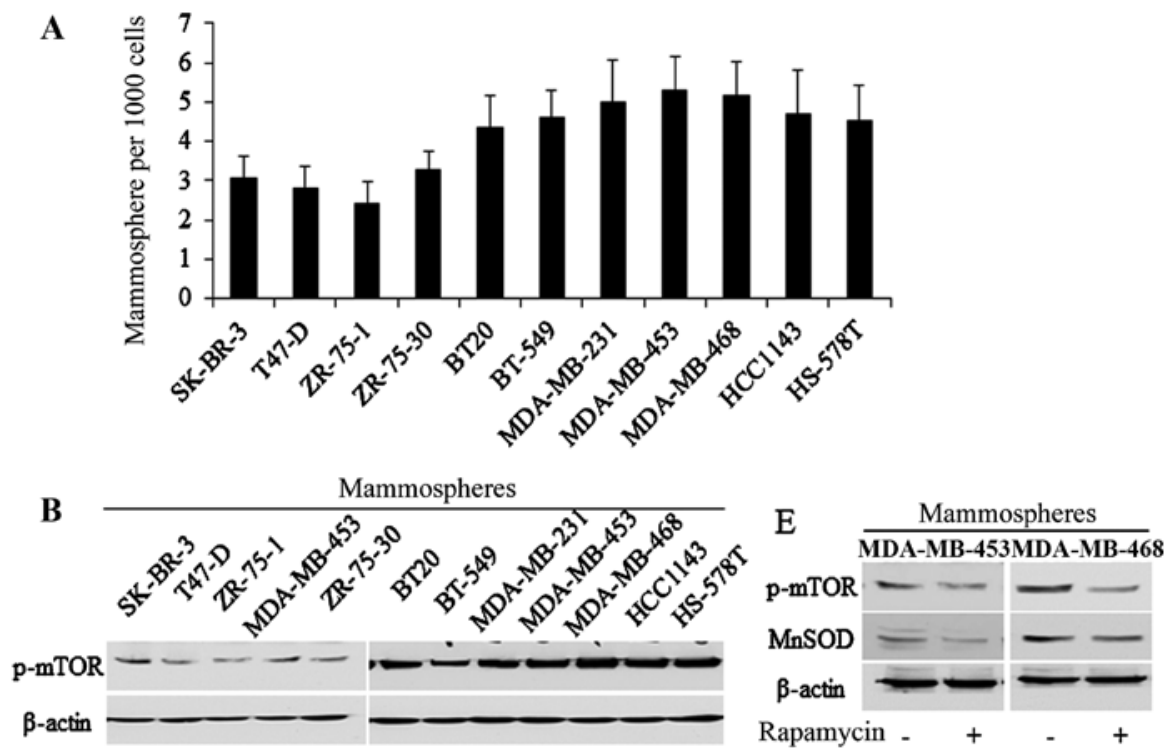

C

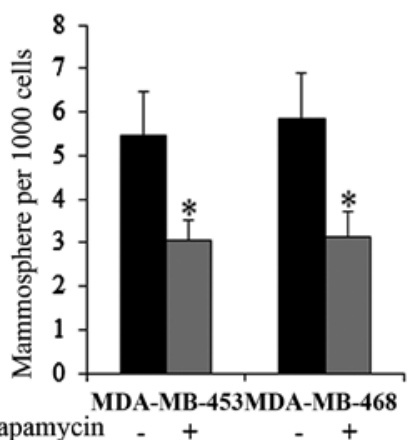

The $1^{\text {st }}$ generation of mammospheres

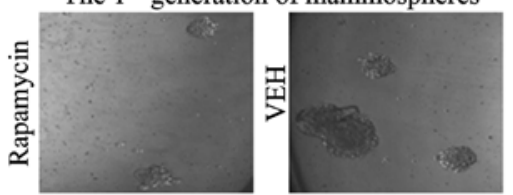

D

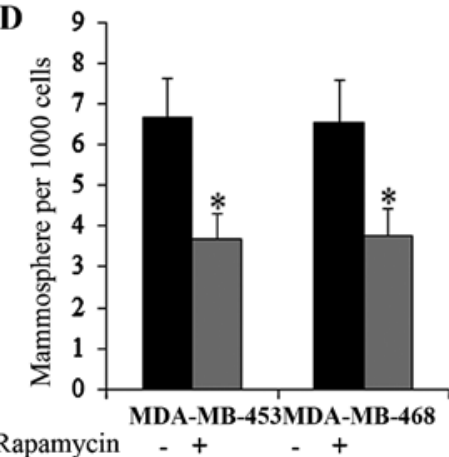

The $2^{\text {nd }}$ generation of mammospheres

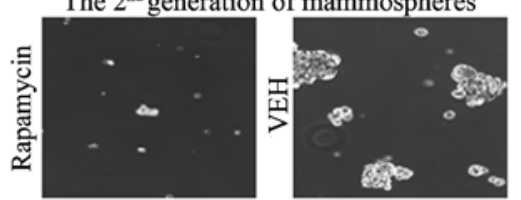

Figure 1. Mammalian target of rapamycin (mTOR) activity is correlated with the self-renewal ability of breast cancer stem cells (BrCSCs). (A) The self-renewal ability of MDA-MB-453 and MDA-MB-468 was much higher than that of stem cells from other breast cancer cell lines. (B) Endogenous p-mTOR expression in multiple breast cancer cell lines, of which MDA-MB-453 and MDA-MB-468 exhibited the strongest mTOR activity. The effects of rapamycin on selfrenewal capacity of BrCSCs were tested, and the cells were incubated at $37^{\circ} \mathrm{C}$ for $12 \mathrm{~h}$ with PBS or $20 \mu \mathrm{M}$ rapamycin, which strongly inhibited the number of mammospheres in the first (C) and second (D) generation. (E) Twenty micrometers of rapamycin inhibited mTOR phosphorylation and manganese superoxide dismutase (MnSOD) activity, correlating with the decreased ability of self-renewal of MDA-MB-453 and MDA-MB-468 compared to the control group." $<<0.01$.

dye (no. 62248) (both from Life Technologies, Thermo Fisher Scientific). In order to study the division modes of CSCs, the mammospheres were disaggregated and seeded in chambers $24 \mathrm{~h}$ prior to staining. Akt-pan (C67E7, no. 4691; Cell Signaling Technology, Inc.) was used to identify the asymmetrically divided stem cells (21), as previously described, and the uneven or asymmetric distribution of pan-Akt was taken to indicate the occurrence of asymmetric division (AD) in CSCs, as previously described $(21,28,29)$.

Dihydroethidium (DHE) staining. DHE (Molecular Probes, Vigorous Inc., Beijing, China) is an oxidative fluorescent dye, and was used to evaluate theROS levels in the cells. ROS production was assessed using a FACSAria flow cytometer. The cells were cultured with $50 \mu \mathrm{M}$ of DHE for $60 \mathrm{~min}$ at $37^{\circ} \mathrm{C}$, and were kept in the dark. The cells were then trypsinized and subjected to flow cytometry at an excitation wavelength of $515 \mathrm{~nm}$, and a waudio videoelength of $600 \mathrm{~nm}$. The ROS-positive cells presented strong red fluorescence, compared to the ROS-negative group.

Statistical analysis. All data in this study were obtained from three independent experiments, and are expressed as the means \pm SD. Statistical analysis was performed using a Student's t-test and $\chi^{2}$ test with SPSS 16.0 for Windows (IBM, Chicago, IL, USA) and Excel 2007 (Microsoft Corporation, Redmond, WA, USA). A P-value $<0.01$ was deemed to indicate a statistically significant difference.

\section{Results}

Activation of $m T O R$ phosphorylation is crucial to the self-renewal ability of HS587-T and MDA-MB-231 stemcells.In 

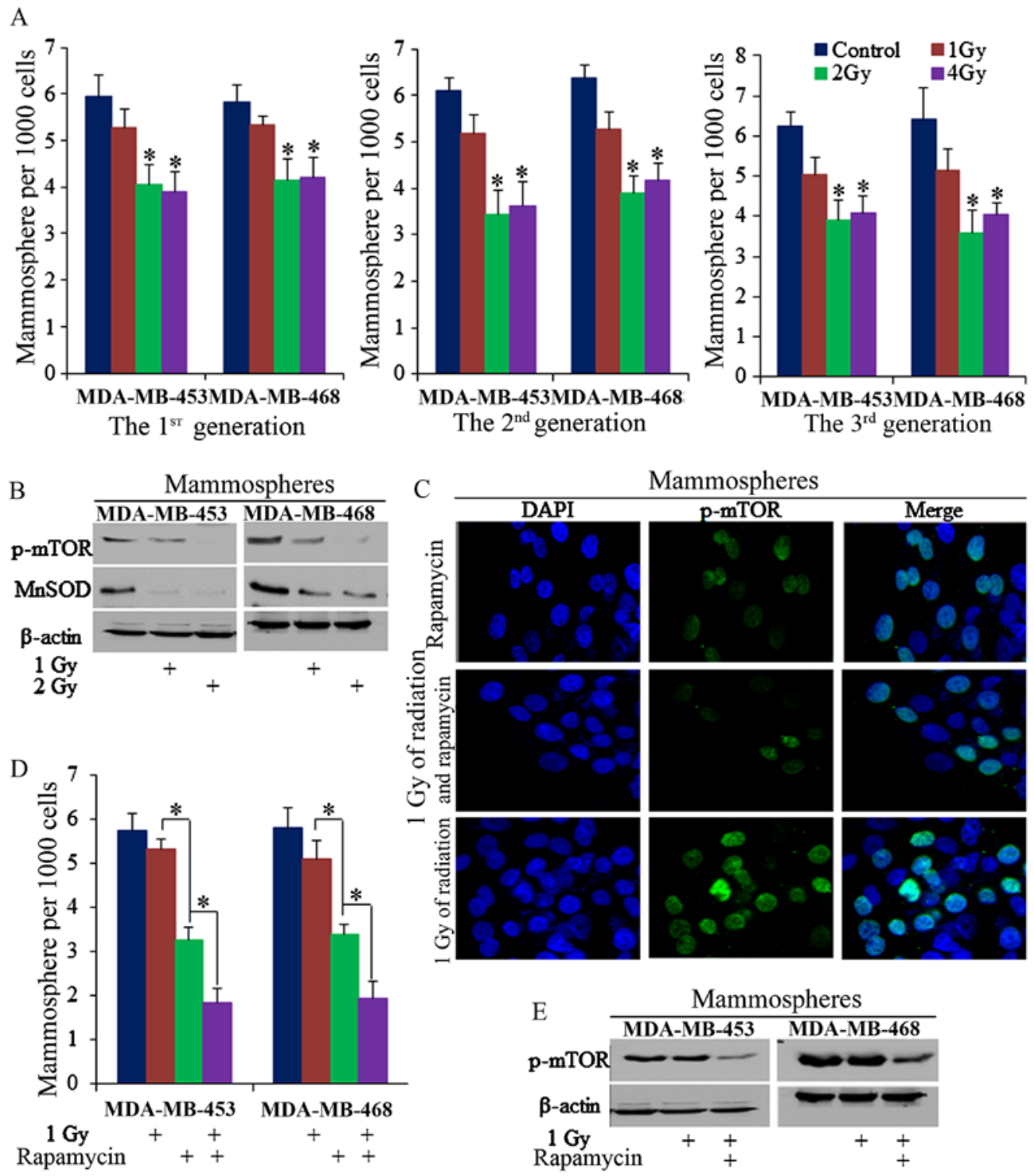

Figure 2. Mammalian target of rapamycin (mTOR) inhibition sensitized breast cancer stem cells (BrCSCs) to the effects of radiation and decreased the number of mammospheres. (A) One gray of radiation failed to suppress the self-renewal of MDA-MB-453 and MDA-MB-468 stem cells in the continuous cultivation of mammospheres. (B and C) Decreased expression of p-mTOR (Ser2481) and manganese superoxide dismutase (MnSOD) in stem cells of MDA-MB-453 and MDA-MB-468 was correlated with the inhibitive functions of radiation. (D) Twenty micrometers of rapamycin effectively sensitized BrCSCs to 1 Gy of radiation, which induced inhibition of self-renewal via synergic suppression of (E) phosphorylated mTOR compared to the control group, ${ }^{*}$ p $<0.01$.

order to identify the roles of mTOR in BrCSCs, we first detected endogenous mTOR activity in multiple breast cancer cell lines, as previously described (30). In breast cancer cell lines SK-BR-3, T47-D, ZR-75-1, ZR-75-30, BT20, BT-549, MDA-MB-231, MDA-MB-453, MDA-MB-468, HCC1143 and HS-578T, the mTOR levels were positively correlated with the self-renewal ability of BrCSCs, and of the cell lines, endogenous mTOR activity was much higher in MDA-MB-453 and MDA-MB-468 stem cells (Fig. 1A and B). Rapamycin (ab120224; Abcam, Shanghai, China) at $20 \mu \mathrm{M}$ significantly decreased the number of mammospheres of 1st and 2nd generations (Fig. 1C and D), while mTOR phosphorylation decreased (Fig. 1E), proving that mTOR plays crucial roles in sustaining the stem cell pool of MDA-MB-453 and MDA-MB-468. On the basis of these results, MDA-MB-468. MDA-MB-453 and MDA-MB-468 stem cells were used subsequently.
mTOR inhibition sensitizes BrCSCs to radiation-induced inhibition of self-renewal. Radiation of $2 \mathrm{~Gy}$ decreased the number of mammospheres of MDA-MB-453 and MDA-MB-468 cells in continuous mammosphere culture (Fig. 2A), with mTOR phosphorylation also being inhibited (Fig. 2B and C). However, 1 Gy of ionizing radiation failed to markedly inhibit the number of mammospheres in these two cell lines, $\mathrm{P}>0.05$ (Fig. 2A), despite the fact that it is known to play a role in the induction of cell apoptosis, as has been previously described (31-33). However, when $1 \mathrm{~Gy}$ of radiation and $20 \mu \mathrm{M}$ rapamycin were combined, 1 Gy of radiation effectively reduced mammosphere formation efficiency (Fig. 2D), and effectively inhibited mTOR phosphorylation in the mammospheres (Fig. 2C and E).

Decreased MnSOD is critical for rapamycin-induced inhibition of self-renewal of BrCSCs treated with ionizing radiation. 
A

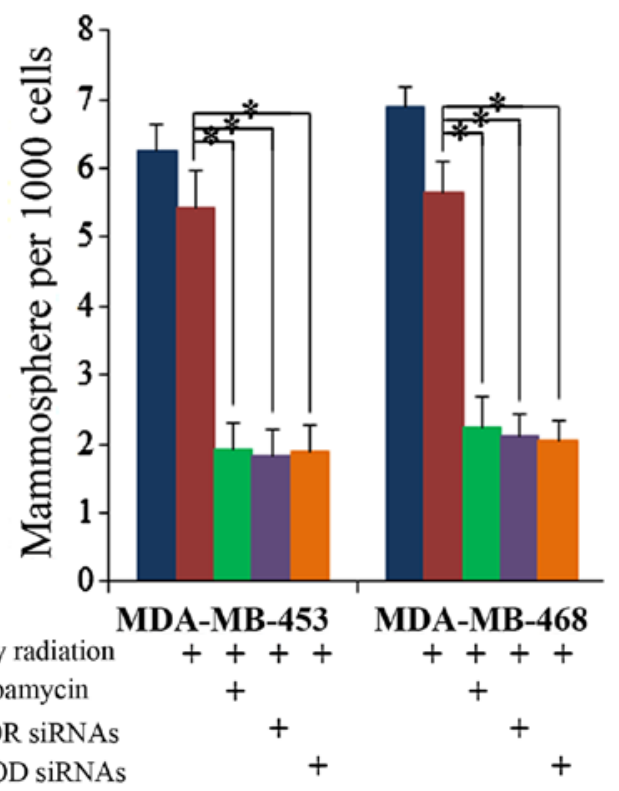

B

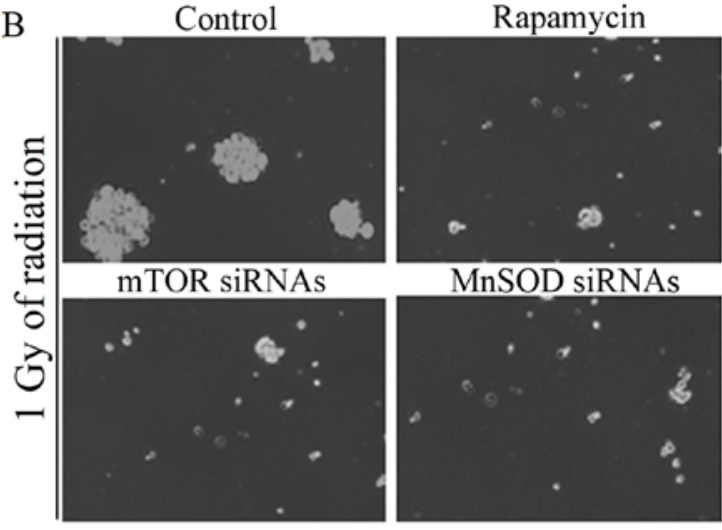

C

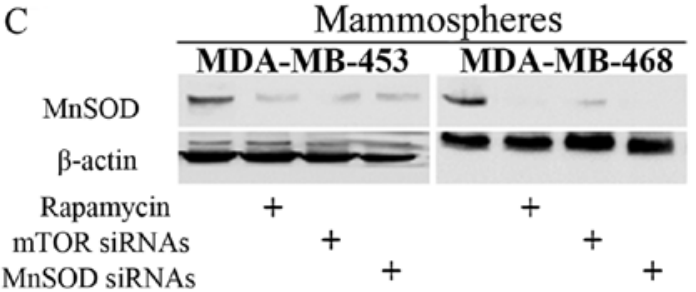

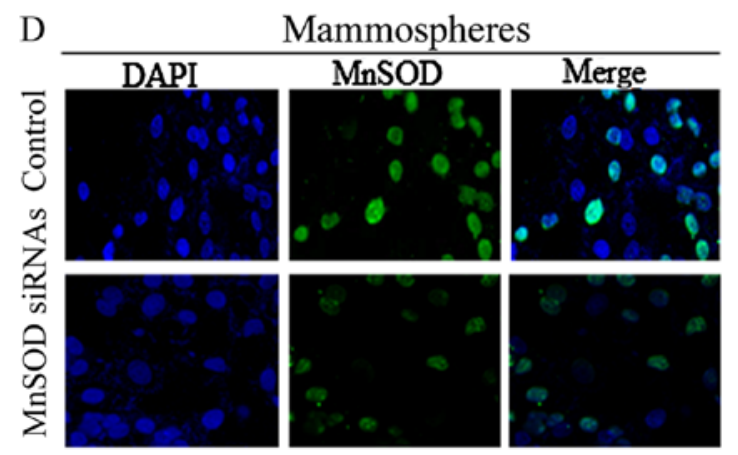

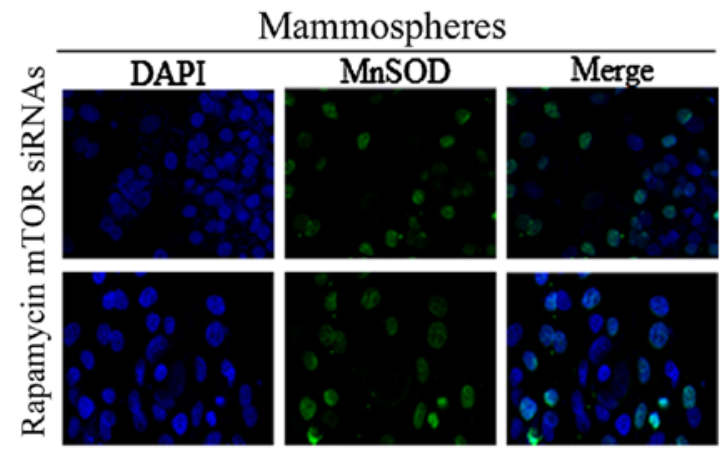

Figure 3. Rapamycin inhibited the self-renewal of stem cells in ionizing radiation-treated breast cancer cells via a decrease in manganese superoxide dismutase (MnSOD) activity. (A and B) Rapamycin exerted similar effects as inhibition of mammalian target of rapamycin (mTOR) and MnSOD, and all sensitized cells to the effects of $1 \mathrm{~Gy}$ of radiation in terms of suppressing stem cell renewal. (C and D) Rapamycin functioned through inhibition of mTOR and MnSOD, which is critical for rapamycin function; rapamycin, mTOR and MnSOD siRNAS decreased the self-renewal of cells compared to the control group, ${ }^{*}$ p $<0.01$.

MnSOD is associated with mTOR activity in stem cells, and we noted that it was suppressed by both rapamycin and radiation (Figs. 1E and 2B). The inhibition of MnSOD sensitized BrCSCs to $1 \mathrm{~Gy}$ of radiation, and we noted that no significant difference between rapamycin- or mTOR siRNA-treated cells was observable (Fig. 3A and B). Both rapamycin and mTOR siRNAs decreased the self-renewal of cells, as did MnSOD siRNAs (Fig. 3C and D).

Akt is required for rapamycin function and sensitization of cells to effects of radiation. Although it is known that mTOR functions through Akt in many ways, it was not known whether mTOR inhibition induces repression of self-renewal through Akt, and the roles which Akt plays in the regulation of mTOR and MnSOD had not previously been explored. In the present study, we found that Akt inhibition decreased the mammosphere formation efficiency (MFE) of BrCSCs (Fig. 4A), and functioned the same way as mTOR inhibition, as had also been previously reported (34-36). The knockdown of Akt by Akt inhibitors (Akti-1/2, ab142088; Abcam, Cambridge, MA, USA) abolished the effects of rapamycin on radiation sensitiza- tion (Fig. 4B). To confirm the role of Akt in rapamycin-induced radiation sensitization, we subsequently used shRNA-Akt1 lentivirals, and similar results were detected compared to the usage of Akt inhibitors (Fig. 4C and D), and downstream MnSOD was not markedly influenced in Akt knockdown cells (Fig. 4E). Thus, we posit that the existence of Akt is required for the rapamycin regulation of MnSOD in the sensitization of BrCSCs to radiation-induced suppression (Fig. 4F).

The existence of Akt is critical for rapamycin-induced asymmetric cell division. mTOR functions via the regulation of MnSOD in normal epithelial stem cell senescence and cancer cell fate (7); however, its roles in CSCs had not previously been discussed. Low ROS activity is one of the most effective biomarkers of a high ability to self-renew, something which has previously been achieved by induction of symmetric cell division (21). Our results showed that ROS activity was upregulated by rapamycin (Fig. 5A), and this was achieved by inhibition of MnSOD, as was also previously suggested $(12,37)$. Moreover, we noted that Akt is also required for rapamycin sensitization of the radiotherapy response and ROS regulation (Fig. 5B). 

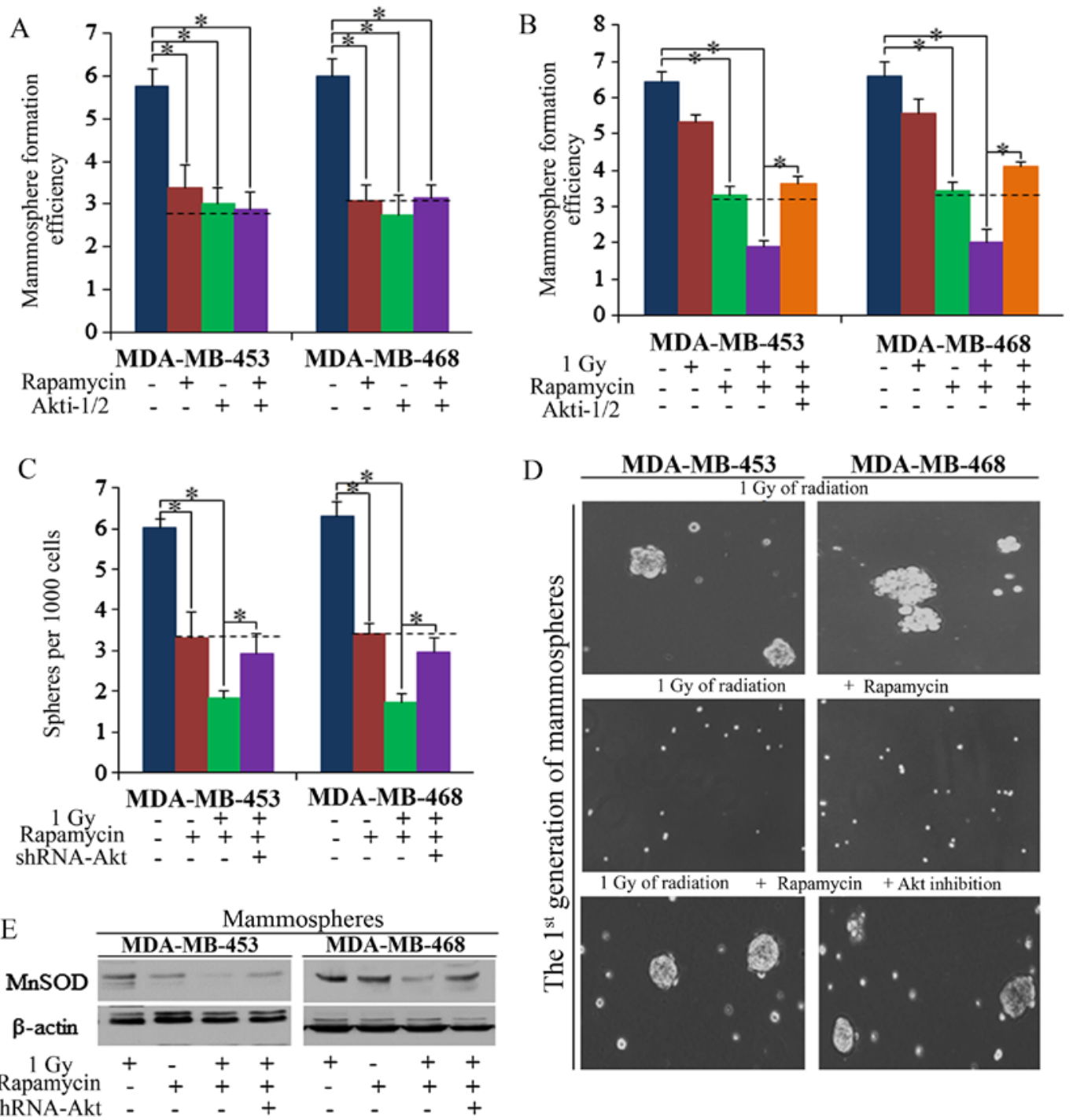

F

1 Gy of radiation and rapamycin (mammospheres)

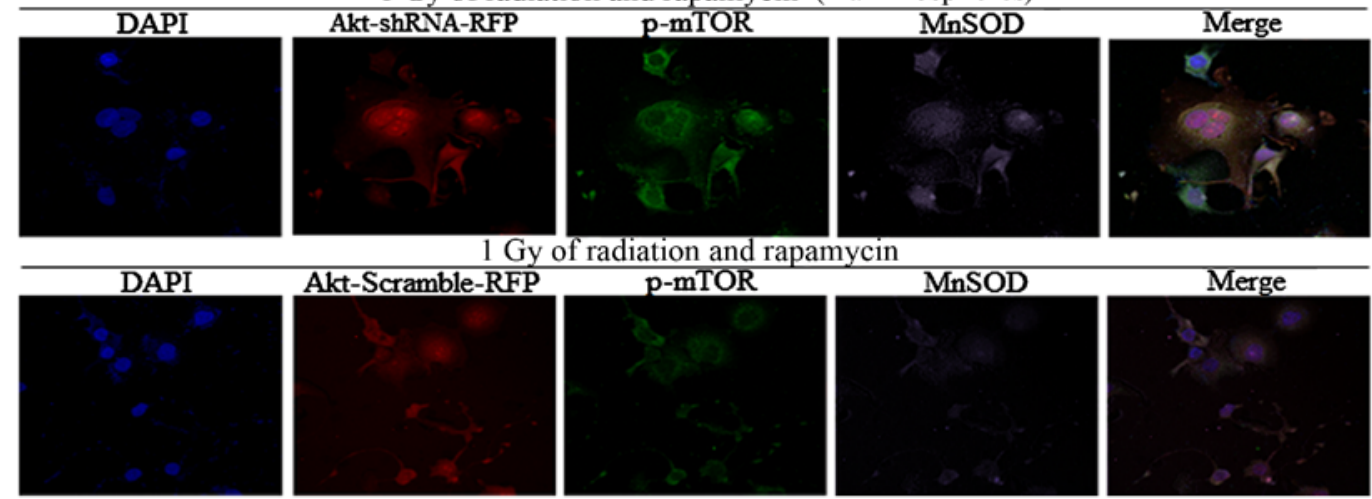

Figure 4. Rapamycin sensitization of low-dose radiation required Akt in cancer cells. (A) Akt inhibitors (Akti-1/2) decreased the number of mammospheres, and did not markedly affect rapamycin function. (B-D) The knockdown of Akt abolished the effects of rapamycin in relation to the induction of radiation sensitization. (E and F) The existence of Akt was required for rapamycin regulation of manganese superoxide dismutase (MnSOD) and sensitization to radiation-induced stem cell repression compared to the control group, ${ }^{*} \mathrm{p}<0.01$.

Increased ROS has been shown to promote the AD of CSCs, and results in repression of self-renewal, helping to sensitize CSCs to the effects of radiation $(21,38,39)$. Asymmetric cell division was recognized through Akt distribution in dividing cells, as was also previously reported $(21,29,40,41)$, and we identified the asymmetrically dividing stem cells by Akt staining at the division stage (Fig. 5C). As shown in Fig. 5D, we found that rapamycin increased the $\mathrm{AD}$ of stem cells, and enhanced the functions of low-dose radiation in relation to the induction of $\mathrm{AD}$, which then resulted in the number of mammospheres 
A

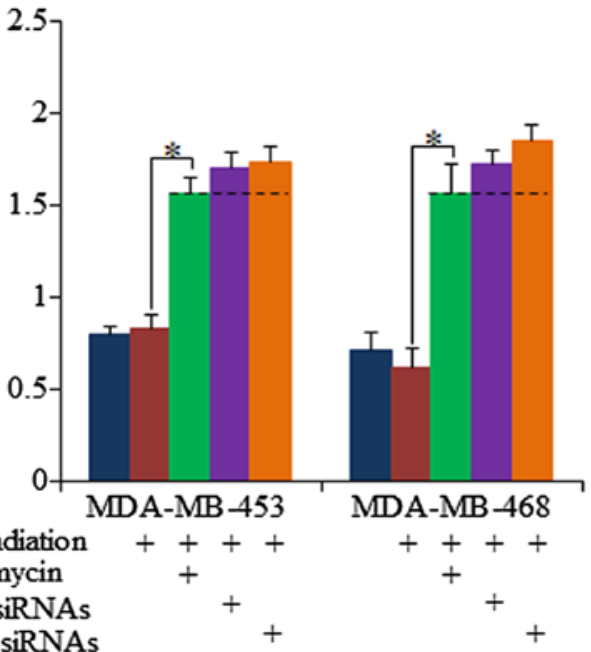

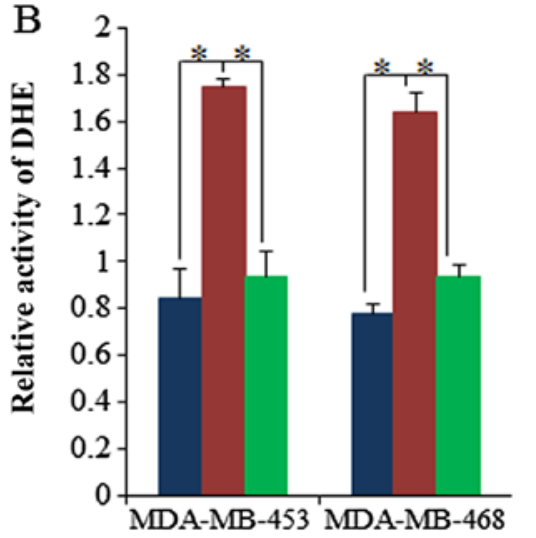

1 Gy Radiation - + + - + + Rapamycin - + + - + +

shRNA-Akt - - + - - +
$\mathrm{C}$
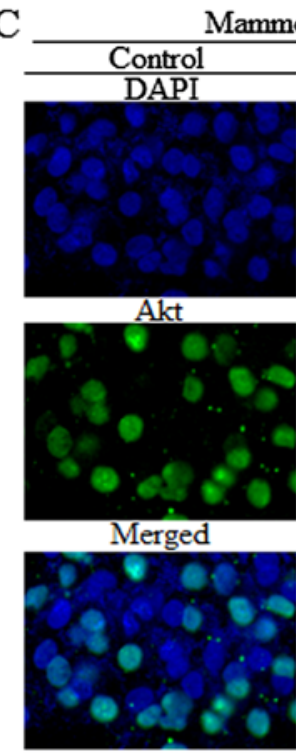
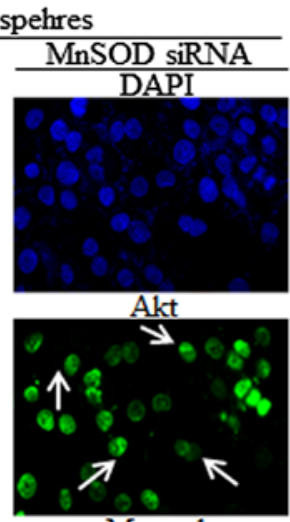

Merged

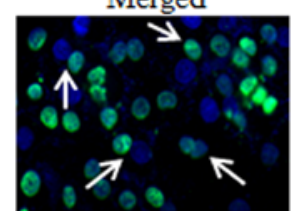

$\mathrm{D}$

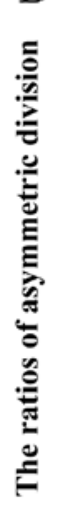

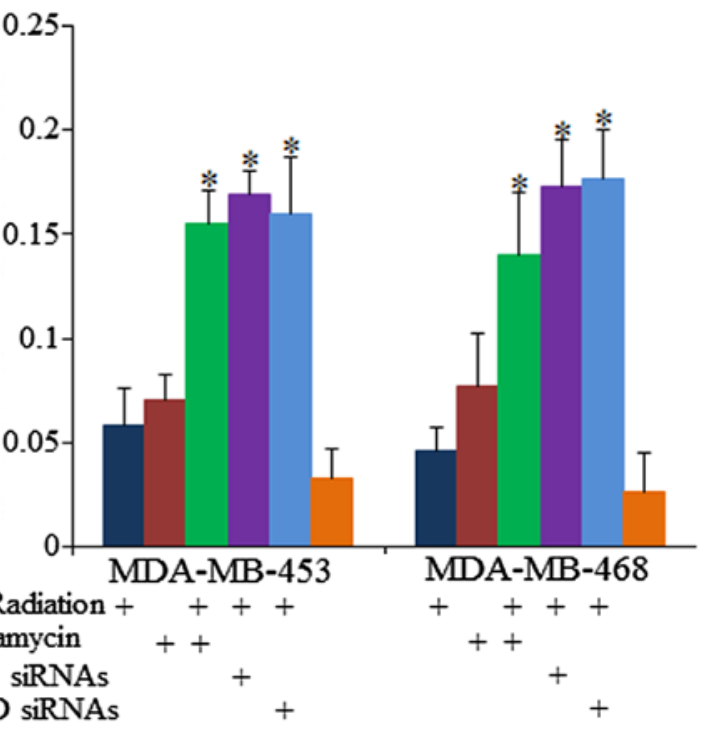

$\mathrm{E}$

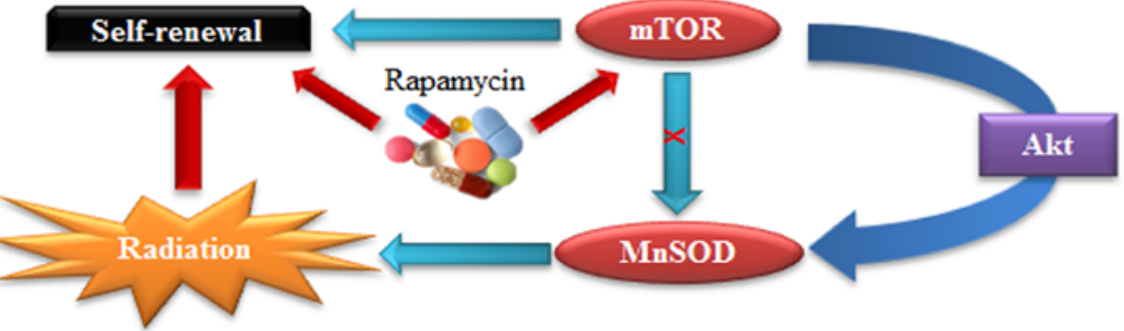

1 Gy Radiation ++++

Rapamycin + + mTOR siRNAs MnSOD siRNAs 
contribute to maintaining the tumor group, therapy resistance and long-term tumor survival and relapse. These stem cells are silent and steady, but, when challenged by various stressors that threaten their numbers, including ionizing radiation, the breast cancer cells begin to generate more stem cells (43), and together with the surviving CSCs repopulate the tumor (44).

Radiotherapy is commonly used in clinical treatments, and new strategies for helping to sensitize breast cancer cells to radiation will thus improve the prospects of patients with worse receptor status (45-48). Sensitization of breast CSCs to the inhibitive effects of chemo-radiotherapies by adjuvant therapy targeting oncogenic pathways represents a step toward improving the outcome of radiation in patients with TNBC. The activation of the mTOR pathway has been recognized as occurring frequently in neoplastic transformation, and our research aims to determine new methods that will improve patient prognosis by inhibiting the expansion of CSCs with mTOR inhibitors (49-51).

Studies of the role of MnSOD in tumorigenesis and cancer progression have yielded conflicting results in relation to cancer risk, prognosis and susceptibility to therapy; however, recent results indicated that MnSOD contributes to the progression of tumors toward aggressive phenotypes by creating a cellular environment conducive to the decrease of ROS in mitochondria in CSCs $(12,52,53)$. Oxidative stress caused by the accumulation of ROS functions in many ways and influences the self-renewal ability of stem cells in cancer (13).

In this study, we examined whether mTOR blockade by rapamycin increased radiation-induced self-renewal inhibition of CSCs in multiple breast cancer cell lines, and our results demonstrated that mTOR activity is closely related to the self-renewal ability of BrCSCs. In mammospheres from MDA-MB-453 and MDA-MB-468 cells, rapamycin repression of mTOR phosphorylation decreased the number of mammospheres, and helped to sensitize the resistant CSCs to low-dose radiation therapy. By using siRNAs targeting mTOR and MnSOD, we confirmed that rapamycin functioned via the mTOR/MnSOD/ROS signaling pathway, and the existence of Akt is necessary for rapamycin induction of $\mathrm{AD}$ of the stem cells in radiation-treated breast cancer. The synergic effects of rapamycin and low-dose radiation induced the AD of stem cells, which then resulted in the decrease of number of mammospheres, and are critical for improved radiotherapy responses in clinical treatment. To the best of our knowledge, for the first time, it has been demonstrated that different doses of radiotherapy cause different outcomes in relation to self-renewal of cells, and that $1 \mathrm{~Gy}$ of radiation did not significantly influence the accumulation of mammospheres of breast cancer, but this effect was improved and strengthened by mTOR inhibition, and we also noted that Akt is required for the sensitization of stem cells to low-dose radiation. Thus, we posit that an in-depth understanding of the interaction of radiation with CSCs has the potential to make radiation even better and more effective.

\section{Acknowledgements}

The authors are grateful for the help and support of the colleagues and staff of the Department of Thyroid and Breast Surgery, and the Department of Obstetrics and Gynecology, the First Affiliated Hospital of Sun Yat-sen University. The authors are also grateful for the guidance from the administra- tors of the central laboratories of the First Affiliated Hospital of Sun Yat-sen University.

\section{References}

1. Kanavos P. The rising burden of cancer in the developing world. Ann Oncol Suppl 8: viii15-viii23.

2. Sun X, Qin S, Fan C, Xu C, Du N and Ren H: Let-7: A regulator of the ER $\alpha$ signaling pathway in human breast tumors and breast cancer stem cells. Oncol Rep 29: 2079-2087, 2013.

3. Assi HA, Khoury KE, Dbouk H, Khalil LE, Mouhieddine TH and El Saghir NS: Epidemiology and prognosis of breast cancer in young women. J Thorac Dis 5 (Suppl 1): S2-S8, 2013.

4. Ohno S, Takanashi M, Sudo K, Ueda S, Ishikawa A, Matsuyama N, Fujita K, Mizutani T, Ohgi T, Ochiya T, et al: Systemically injected exosomes targeted to EGFR deliver antitumor microRNA to breast cancer cells. Mol Ther 21: 185-191, 2013.

5. Yao Y, Hu J, Shen Z, Yao R, Liu S, Li Y, Cong H, Wang X, Qiu W and Yue L: MiR-200b expression in breast cancer: a prognostic marker and act on cell proliferation and apoptosis by targeting Sp1. J Cell Mol Med 19: 760-769, 2015.

6. Liu FJ, Wang XB and Cao AG: Screening and functional analysis of a differential protein profile of human breast cancer. Oncol Lett 7: 1851-1856, 2014.

7. Iglesias-Bartolome R, Patel V, Cotrim A, Leelahavanichkul K, Molinolo AA, Mitchell JB and Gutkind JS: mTOR inhibition prevents epithelial stem cell senescence and protects from radiation-induced mucositis. Cell Stem Cell 11: 401-414, 2012.

8. Castilho RM, Squarize CH, Chodosh LA, Williams BO and Gutkind JS: mTOR mediates Wnt-induced epidermal stem cell exhaustion and aging. Cell Stem Cell 5: 279-289, 2009.

9. Martelli AM, Evangelisti C, Follo MY, Ramazzotti G, Fini M, Giardino R, Manzoli L, McCubrey JA and Cocco L: Targeting the phosphatidylinositol 3-kinase/Akt/mammalian target of rapamycin signaling network in cancer stem cells. Curr Med Chem 18: 2715-2726, 2011.

10. Matsubara S, Ding Q, Miyazaki Y, Kuwahata T, Tsukasa K and Takao S: mTOR plays critical roles in pancreatic cancer stem cells through specific and stemness-related functions. Sci Rep 3: 3230,2013

11. Sun X, Jiao X, Pestell TG, Fan C, Qin S, Mirabelli E, Ren H and Pestell RG: MicroRNAs and cancer stem cells: the sword and the shield. Oncogene 33: 4967-4977, 2014.

12. Hart PC, Mao M, de Abreu ALP, Ansenberger-Fricano K, Ekoue DN, Ganini D, Kajdacsy-Balla A, Diamond AM, Minshall RD, Consolaro ME, et al: MnSOD upregulation sustains the Warburg effect via mitochondrial ROS and AMPK-dependent signalling in cancer. Nat Commun 6: 6053, 2015.

13. Dhar SK, Tangpong J, Chaiswing L, Oberley TD and St Clair DK: Manganese superoxide dismutase is a p53-regulated gene that switches cancers between early and advanced stages. Cancer Res 71: 6684-6695, 2011.

14. Hirsch HA, Iliopoulos D and Struhl K: Metformin inhibits the inflammatory response associated with cellular transformation and cancer stem cell growth. Proc Natl Acad Sci USA 110: 972-977, 2013.

15. Rupaimoole R, Han HD, Lopez-Berestein G and Sood AK: MicroRNA therapeutics: principles, expectations, and challenges. Chin J Cancer 30: 368-370, 2011.

16. Bao S, Wu Q, McLendon RE, Hao Y, Shi Q, Hjelmeland AB, Dewhirst MW, Bigner DD and Rich JN: Glioma stem cells promote radioresistance by preferential activation of the DNA damage response. Nature 444: 756-760, 2006.

17. Phillips TM, McBride WH and Pajonk F: The response of CD $24^{-/ \text {low }} / \mathrm{CD} 44^{+}$breast cancer-initiating cells to radiation. J Natl Cancer Inst 98: 1777-1785, 2006.

18. Woodward WA, Chen MS, Behbod F, Alfaro MP, Buchholz TA and Rosen JM: WNT/beta-catenin mediates radiation resistance of mouse mammary progenitor cells. Proc Natl Acad Sci USA 104: 618-623, 2007.

19. Zielske SP, Spalding AC, Wicha MS and Lawrence TS: Ablation of breast cancer stem cells with radiation. Transl Oncol 4: 227-233, 2011.

20. Diehn M, Cho RW, Lobo NA, Kalisky T, Dorie MJ, Kulp AN, Qian D, Lam JS, Ailles LE, Wong M, et al: Association of reactive oxygen species levels and radioresistance in cancer stem cells. Nature 458: 780-783, 2009. 
21. Dey-Guha I, Wolfer A, Yeh ACG, G Albeck J, Darp R, Leon E, Wulfkuhle J, Petricoin EF III, Wittner BS and Ramaswamy S: Asymmetric cancer cell division regulated by AKT. Proc Nat Acad Sci USA 108: 12845-12850, 2011.

22. Pajonk F, Vlashi E and McBride WH: Radiation resistance of cancer stem cells: the 4 R's of radiobiology revisited. Stem Cells 28: 639-648, 2010.

23. Rycaj $\mathrm{K}$ and Tang DG: Cancer stem cells and radioresistance. Int J Radiat Biol 90: 615-621, 2014.

24. Delort L, Perrier S, Dubois V, Billard H, Mracek T, Bing C, Vasson MP and Caldefie-Chézet F: Zinc- $\alpha 2$-glycoprotein: a proliferative factor for breast cancer? In vitro study and molecular mechanisms. Oncol Rep 29: 2025-2029, 2013.

25. Sun X, Tang SC, Xu C, Wang C, Qin S, Du N, Liu J, Zhang Y, Li X, Luo G, et al: DICER1 regulated let-7 expression levels in p53-induced cancer repression requires cyclin D1. J Cell Mol Med 19: 1357-1365, 2015.

26. Oliveras-Ferraros C,CufíS, Vazquez-Martin A,Torres-GarciaVZ, Del Barco S, Martin-Castillo B and Menendez JA: Micro(mi) RNA expression profile of breast cancer epithelial cells treated with the anti-diabetic drug metformin: induction of the tumor suppressor miRNA let-7a and suppression of the TGF $\beta$-induced oncomiR miRNA-181a. Cell Cycle 10: 1144-1151, 2011.

27. Sun Y, Wang Y, Fan C, Gao P, Wang X, Wei G and Wei J: Estrogen promotes stemness and invasiveness of ER-positive breast cancer cells through Glil activation. Mol Cancer 13: 137, 2014.

28. Henle SJ, Wang G, Liang E, Wu M, Poo MM and Henley JR: Asymmetric PI $(3,4,5) \mathrm{P} 3$ and Akt signaling mediates chemotaxis of axonal growth cones. J Neurosci 31: 7016-7027, 2011

29. Chung CY, Potikyan G and Firtel RA: Control of cell polarity and chemotaxis by Akt/PKB and PI3 kinase through the regulation of PAKa. Mol Cell 7: 937-947, 2001.

30. Chavez KJ, Garimella SV and Lipkowitz S: Triple negative breast cancer cell lines: one tool in the search for better treatment of triple negative breast cancer. Breast Dis 32: 35-48, 2010.

31. Mirzayans R, Andrais B, Scott A, Wang YW and Murray D: Ionizing radiation-induced responses in human cells with differing TP53 status. Int J Mol Sci 14: 22409-22435, 2013.

32. Cataldi A, Di Giacomo V, Rapino M, Zara S and Rana RA Ionizing radiation induces apoptotic signal through protein kinase C $\delta$ (delta) and survival signal through Akt and cyclicnucleotide response element-binding protein (CREB) in Jurkat T cells. Biol Bull 217: 202-212, 2009.

33. Landsverk KS, Lyng $\mathrm{H}$ and Stokke T: The response of malignant B lymphocytes to ionizing radiation: cell cycle arrest, apoptosis and protection against the cytotoxic effects of the mitotic inhibitor nocodazole. Radiat Res 162: 405-415, 2004.

34. McDermott SP and Wicha MS: Targeting breast cancer stem cells. Mol Oncol 4: 404-419, 2010.

35. Suman S, Das TP and Damodaran C: Silencing NOTCH signaling causes growth arrest in both breast cancer stem cells and breast cancer cells. Br J Cancer 109: 2587-2596, 2013.

36. Lombardo Y, Filipović A, Molyneux G, Periyasamy M, Giamas G, Hu Y, Trivedi PS, Wang J, Yagüe E, Michel L and Coombes RC: Nicastrin regulates breast cancer stem cell properties and tumor growth in vitro and in vivo. Proc Natl Acad Sci USA 109: 16558-16563, 2012.

37. Li Z, Shi K, Guan L, Cao T, Jiang Q, Yang Y and Xu C: ROS leads to MnSOD upregulation through ERK2 translocation and p53 activation in selenite-induced apoptosis of NB4 cells. FEBS Lett 584: 2291-2297, 2010.
38. Shi X, Zhang Y, Zheng J and Pan J: Reactive oxygen species in cancer stem cells. Antioxid Redox Signal 16: 1215-1228, 2012.

39. Ito K and Suda T: Metabolic requirements for the maintenance of self-renewing stem cells. Nat Rev Mol Cell Biol 15: 243-256, 2014.

40. Neumüller RA and Knoblich JA: Dividing cellular asymmetry: asymmetric cell division and its implications for stem cells and cancer. Genes Dev 23: 2675-2699, 2009.

41. Dey-Guha I, Alves CP, Yeh AC, Salony, Sole X, Darp R and Ramaswamy S: A mechanism for asymmetric cell division resulting in proliferative asynchronicity. Mol Cancer Res 13: 223-230, 2015.

42. Jemal A, Bray F, Center MM, Ferlay J, Ward E and Forman D: Global cancer statistics. CA Cancer J Clin 61: 69-90, 2011.

43. Lagadec C, Vlashi E, Della Donna L, Dekmezian C and Pajonk F: Radiation-induced reprogramming of breast cancer cells. Stem Cells 30: 833-844, 2012.

44. Printz C: Radiation treatment generates therapy-resistant cancer stem cells from less aggressive breast cancer cells. Cancer 118: $3225,2012$.

45. Wu L, Shao L, Li M, Zheng J, Wang J, Feng W, Chang J, Wang Y, Hauer-Jensen M and Zhou D: BMS-345541 sensitizes MCF-7 breast cancer cells to ionizing radiation by selective inhibition of homologous recombinational repair of DNA double-strand breaks. Radiat Res 179: 160-170, 2013

46. Lai CH, Chang CS, Liu HH, Tsai YS, Hsu FM, Yu YL, Lai CK, Gandee L, Pong RC, Hsu HW, et al: Sensitization of radioresistant prostate cancer cells with a unique cytolethal distending toxin. Oncotarget 5: 5523-5534, 2014.

47. Gu Q, He Y, Ji J, Yao Y, Shen W, Luo J, Zhu W, Cao H, Geng Y, $\mathrm{Xu} \mathrm{J}$, et al: Hypoxia-inducible factor $1 \alpha(\mathrm{HIF}-1 \alpha)$ and reactive oxygen species (ROS) mediates radiation-induced invasiveness through the SDF-1 $\alpha /$ CXCR4 pathway in non-small cell lung carcinoma cells. Oncotarget 6: 10893-10907, 2015.

48. Sun X, Jiang S, Liu J, Wang H, Zhang Y, Tang SC, Wang J, Du N, Xu C, Wang C, et al: MiR-208a stimulates the cocktail of SOX2 and $\beta$-catenin to inhibit the let-7 induction of self-renewal repression of breast cancer stem cells and formed miR208a/let-7 feedback loop via LIN28 and DICER1. Oncotarget 6: 32944-32954, 2015.

49. Cruceru ML, Neagu M, Demoulin J-B and Constantinescu SN: Therapy targets in glioblastoma and cancer stem cells: lessons from haematopoietic neoplasms. J Cell Mol Med 17: 1218-1235, 2013.

50. Bartalucci N, Tozzi L, Bogani C, Martinelli S, Rotunno G, Villeval JL and Vannucchi AM: Co-targeting the PI3K/mTOR and JAK2 signalling pathways produces synergistic activity against myeloproliferative neoplasms. J Cell Mol Med 17: 1385-1396, 2013

51. Rosner M, Hanneder M, Siegel N, Valli A, Fuchs C and Hengstschlager M: The mTOR pathway and its role in human genetic diseases. Mutat Res 659: 284-292, 2008.

52. Britschgi A, Bill A, Brinkhaus H, Rothwell C, Clay I, Duss S, Rebhan M, Raman P, Guy CT, Wetzel K, et al: Calcium-activated chloride channel ANO1 promotes breast cancer progression by activating EGFR and CAMK signaling. Proc Natl Acad Sci USA 110: E1026-E1034, 2013.

53. Zhou D, Shao L and Spitz DR: Reactive oxygen species in normal and tumor stem cells. Adv Cancer Res 122: 1-67, 2014 